\title{
Antibiotic resistance in extra intestinal pathogenic Escherichia Coli
}

\begin{abstract}
The increase in bacterial antibiotic resistance is a major concern in India. Infections due to multidrug resistance extra intestinal pathogenic $E$. coli unquestionably have substantial effects on morbidity and mortality. The aim of this study was to conduct a systematic review of studies reporting on the prevalence of antibiotic resistance in E. coli in different part of the India.
\end{abstract}

Volume 4 Issue 4 - 2017

\author{
Arindam Chakraborty \\ Department of Microbiology, Motilala Nehru Medical College, \\ India \\ Correspondence: Arindam Chakraborty, Department of \\ Microbiology, Assistant Professor, Motilala Nehru Medical \\ College, Allahabad, Utter Pradesh, India, \\ Email rindamchakraborty@hotmail.com
}

Received: February 21, 2017 | Published: April 06, 2017

\section{Introduction}

In recent years, extra intestinal $E$. coli infection and a high level of drug resistance among such ExPEC is often seen in the World. The treatment of $E$. coli infections is increasingly becoming difficult because of the multi-drug resistance exhibited by the organism. As multi- drug resistance has spread, we are already coming across infections that cannot be cured with any available antibiotics. Extended spectrum $\beta$-lactamase (ESBL) producing E. coli has spread as a major cause of hospital-acquired infections, as well as infections in outpatient settings. ${ }^{1}$ Genes that encode ESBLs are often found on large plasmids that also carry genes for resistance to other antibiotics. ${ }^{2}$ Beginning in the 1990 s, the frequency of resistance to fluoroquinolone antibiotics, including ciprofloxacin, levofloxacin, moxifloxacin, norfloxacin, and nalidixic acid in E. coli has increased worldwide. ${ }^{3}$ Administration of ciprofloxacin or other fluoroquinolones is a risk factor for isolation of resistant strains of $E$. coli from patients undergoing long-term hospital care, and resistance is associated with treatment failure. In addition, multi-resistance, defined as resistance to norfloxacin in addition to two or three other antibiotics, has also increased. ${ }^{3,4}$ Several reports have indicated that quinolone resistance in uropathogenic $E$. coli is associated with decreased prevalence or expression of virulence factors compared to quinolone- susceptible strains. ${ }^{5,6}$ Vila suggested that a possible reason for this is that virulence genes could be lost concomitant with a mutation at codon 83 of the gyrA gene, which affects super coiling of DNA, leading to changes in gene expression. Another reason is that with exposure to quinolones and development of resistance to these agents, there is a concomitant increase in the deletion and transposition of pathogenicity islands (PAIs). ${ }^{7}$ Soto et al. ${ }^{6}$ found that uropathogenic E. coli strains incubated with sub inhibitory concentrations of ciprofloxacin showed partial or total loss of virulence genes encoded within PAIs. ${ }^{6}$

In a study from India have shown high degree of resistance pattern to commonly used antibiotics such as ampicillin, piperacillin, ciprofloxacin, norfloxacin. However, around $50 \%$ isolates were resistant to ceftazidime. They also observed that $25 \%$ isolates were resistant to piperacillin/tazobactam and around $35 \%$ of the isolates were resistant to cefoperazone/sulbactam which is quite alarming, higher sensitivity was observed in Amikacin (84\%) and Nitrofurantoin $(89 \%)$. Among all the antibiotics they tested highest degree of sensitivity were observed with ertapenem (97\%) and other carbapenem group of drugs. ${ }^{8}$ A study by Banu et al. ${ }^{9}$ in patients with $E$. coli infections found around $96 \%$ ampicillin resistance, $74 \%$ co-trimoxazole, $44 \%$ ciprofloxacin, $56 \%$ gentamicin and $35 \%$ amikacin resistance respectively ${ }^{9}$ Yet another study by Sharma et al in Mangalore also reported high prevalence of antibiotic resistance among the E.coli isolates. ${ }^{10}$

In a study from India have reported a high rate of genotypically ESBL positive isolates $(70 \%)$, which indicated a high prevalence of ESBL producers in their setup compared to western figure. They have found CTX-M as the most predominant type of plasmid among the ESBL producers (88\%) and only $19 \%$ were TEM and presence of SHV type was seen in very few isolates (2\%). Among the combinations of ESBL genes detected, only $6 \%$ isolates were found to carry both CTX-M and SHV among the subtypes CTX-M15 was the most predominant $(92 \%)$ among the isolates. ${ }^{11}$ Zaniani et al. ${ }^{12}$ from Iran have also reported that $44 \%$ of their isolates to be ESBL positive among them $15 \%$ were positive with SHV and $21 \%$ were positive with TEM. ${ }^{12}$ In India Goyal et al. ${ }^{13}$ used specific primers for TEM, SHV and CTX-M and showed that $82(75.2 \%)$ of 109 ESBL isolates could be typed for one or more genes which is comparable to our study. Two or more ESBL genes were present in $57.3 \%$ of typeable isolates. The bla CTX-M was the most common and was present either alone or in combination with other ESBL type(s), their finding support the hypothesis that CTX-M is emerging as the dominant ESBL type in clinical isolates. ${ }^{13}$ CTX-M-15 is known to be an ESBL that has peculiar association with community-onset $E$. coli infections. Ensor et al. ${ }^{14}$ have shown that in India bla CTX- M- 15 are the most prevalent than any other type and it is widely distributed in both North and South India. They also reported that the Indian population represented a significant reservoir and source of bla CTX- M- $15 .{ }^{14}$

In a study from India reported that around $10 \%$ of the ExPEC isolates were carbapenemase producers. ${ }^{15}$ Several other studies from India have also reported the prevalence rate of $8-10 \%$ of enterobacteriaceae isolates being carbapenemase producers. ${ }^{16,17}$ The problem with carbapenemase producing isolates is their unrivalled broad- spectrum resistance profile. These strains are usually resistant to $\beta$ - lactams, aminoglycosides and fluoroquinolones. However, they usually remain susceptible to polymyxins. No extended survey with a series of human infections with carbapenemase positive isolates has been performed to determine the optimal treatment. The only 
therapeutic alternative may be the therapeutic administration of polymyxins, which have recently been shown to be efficient for treating multidrug - resistance gram- negative bacilli. In any case, these molecules should not be used as monotherapy and rapid determination of MICs of aminoglycosides by MIC methods (not disk diffusions) may help to choose an aminoglycoside molecule that may have kept some activity. Hence we recommended aminoglycoside and polymyxin combination would be the ideal therapy for such infection.

\section{Conclusion}

The result of this literature review underlines the necessity to establish mechanism for periodic assessment of antimicrobial susceptibility and its determinant in different population from different part of India. Such a monitoring of the antibiotic resistance pattern will help the microbiology laboratories and hospitals to make proper antibiotic policies to limit the spread of MDR E. coli strains among the population.

\section{Conflicts of Interest}

The authors declare no conflict of interest.

\section{Acknowledgments}

None.

\section{Funding}

None.

\section{References}

1. Oteo J, Lázaro E, de Abajo FJ, et al. Antimicrobial- resistant invasive Escherichia coli, Spain. Emerg Infect Dis. 2005;11(4):546-553.

2. Livermore DM, Woodford $N$. The $\beta$ - lactamase threat in Enterobacteriaceae, Pseudomonas and Acinetobacter. Trends Microbiol 2006;14(9):413-420.

3. Goettsch W, van Pelt W, Nagelkerke N, et al. Increasing resistance to fluoroquinolones in Escherichia coli from urinary tract infections in The Netherlands. J Antimicrob Chemother. 2000;46(2):223-228.

4. Hopkins KL, Davies RH, Threlfall EJ. Mechanisms of quinolone resistance in Escherichia coli and Salmonella: recent developments. Int J Antimicrob Agents. 2005;25(5):358-373.

5. Horcajada JP, Soto S, Gajewski A, et al. Quinolone- resistant uropathogenic Escherichia coli strains from phylogenetic group B 2 have fewer virulence factors than their susceptible counterparts. J Clin Microbiol. 2005;43(6):2962-2964.
6. Soto SM, Jimenez de Anta MT, Vila J. Quinolones inducepartial or total loss of pathogenicity islands in uropathogenic Escherichia coli by SOSdependent or - independent pathways, respectively. Antimicrob Agents Chemother. 2006;50(2):649-653.

7. Vila J, Simon K, Ruiz J, et al. Are quinolone resistant uropathogenic Escherichia coli less virulent? J Infect Dis. 2002;186(7):1039-1042.

8. Chakraborty Arindam, Adhikari Prabha, Shenoy Shalini, et al. JCDRExtra- intestinal $\beta$ - lactamase Producing Escherichia coli Infection- an Emerging Infection in a South Indian Tertiary Care Hospital. Journal of Clinical and diagnostic Research. 2012;6(7):1210-1214.

9. Banu A, Kabbin SJ, Anand M. Extraintestinal Infections due to Escherichia Coli: An Emerging Issue. JCDR. 2011;5(3):486- 490.

10. Sharma S, Bhat GK, Shenoy S. Virulence Factors and Drug Resistance in Escherichia Coli isolated from extraintestinal infection. Indian J Med Microbiol. 2007;25(4):369-373.

11. Chakraborty Arindam, Adhikari Prabha, Shenoy Shalini, et al. Clinical significance and phylogenetic background of Extended Spectrum $\beta$ lactamase producing Escherichia coli isolates from extra- intestinal infections. J Infect Public Health. 2015;8(4):248-253.

12. Zaniani RF, Meshkat Z, Nasab NM, et al. The Prevalence of TEM and SHV Genes among Extended- Spectrum Beta- Lactamases Producing Escherichia Coli and Klebsiella Pneumoniae. Iranian J Basic Med Sci. 2012;15(1):654-660.

13. Goyal A, Prasad KN, Prasad A, et al. Extended spectrum $\beta$ - lactamases in Escherichia coli \& Klebsiella pneumoniae \& associated risk factors. Indian J Med Res. 2009;129(6):695-700.

14. VM Ensor, M Shahid, JT Evans, et al. Occurrence, prevalence and genetic environment of CTX- M b- lactamases in Enterobacteriaceae from Indian hospitals. J Antimicrob Chemother. 2006;58(6):1260-1263.

15. Chakraborty A, Adhikari P, Shenoy S, et al. Expression of ESBL, MBL and AmpC $\beta$ lactamases by extra intestinal Escherichia coli isolates:correlation with treatment and clinical outcome. J Microbiol Infect Dis. 2013;3(4):150-156.

16. Bora A, Ahmed GU, Hazarika NK, et al. Incidence of blaNDM- 1 gene in Escherichia coli isolates at a tertiary care referral hospital in Northeast India. Indian J Med Microbiol. 2013;31(3):250-256.

17. Deshpande P, Rodrigues C, Shetty A, et al. New Delhi metallo- beta lactamase (NDM-1) in Enterobacteriaceae: treatment options with carbapenems compromised. J Assoc Physicians India. 2010;58: 147149 